\title{
CARACTERIZACIÓN DE PRÁCTICAS DE AUTOCUIDADO Y PERCEPCIÓN DEL RIESGO LABORAL EN TRABAJADORES DE UN GRUPO DE EMPRESAS DE CALZADO UBICADAS EN BUCARAMANGA, COLOMBIA PostCOVID-19* Characterization of self-care practices and perception of
occupational risk in workers of a group of footwear companies
located in Bucaramanga, Colombia PostCOVID-19
}

*Artículo de investigación con enfoque mixto y corte transversal. Es resultado de un trabajo de investigación culminado del programa de Ingeniería Industrial, de la Universidad de Investigación y Desarrollo (UDI), vinculado con la línea de Gestión Industrial, dentro del Grupo de Investigación SINERGIA, de la Universidad de Investigación y Desarrollo (UDI). Fue financiado con recursos propios de los investigadores. Dirección: calle 9 n. 23-55 (Bucaramanga, Colombia).

\begin{abstract}
Yennifer Rojas Caceres
Angie Lisbeth Ordoñez Ortiz (D)

Profesional en Ingeniería Industrial, Universidad de Investigación y Desarrollo (UDI). Grupo de Investigación

SINERGIA, Universidad de Investigación y Desarrollo (Bucaramanga, Colombia).

Correo electrónico: yrojas8@udi.edu.co // aordonez4@udi.edu.co.

Cindy Tatiana Daza Ríos (D)

Bacterióloga y Laborista clínico de la Universidad Industrial de Santander, Especialista en Salud Ocupacional de la Universidad Manuela Beltrán y Magister en Desarrollo Sostenible y Medio Ambiente de la Universidad de Manizales. Docente investigadora del Grupo de Investigación SINERGIA, Universidad de Investigación y Desarrollo -UDI (Bucaramanga, Colombia). Correo electrónico: cdaza1@udi.edu.co
\end{abstract}

| Recibido/Received: 14/04/2021 • Aprobado/Approved: 29/06/2021 • Publicado/Published: 30/07/2021

\section{Resumen}

A partir del presente estudio se buscó caracterizar las prácticas de autocuidado y la percepción del riesgo laboral, entre los trabajadores de un grupo de empresas de calzado, ubicadas en Bucaramanga, Colombia. Lo anterior, se realizó por medio de un diario de campo y de un cuestionario, el cual fue aplicado a 46 trabajadores de 9 empresas de calzado. Los resultados obtenidos dejan ver que los trabajadores tienen un nivel bajo en cuanto a la aplicación de prácticas de autocuidado, e igualmente presentan un nivel bajo respecto de la percepción del riesgo. De acuerdo con lo anterior, se considera necesario generar estrategias que aumenten las prácticas de autocuidado y la percepción del riesgo entre dichos trabajadores; ello, con el objetivo de mejorar el bienestar de estos.

Palabras clave: bienestar laboral, condición de trabajo, percepción, salud, estilo de vida.

\section{Abstract}

The study sought to characterize self-care practices and the perception of occupational risk in workers of a group of footwear companies located in Bucaramanga, Colombia, by means of a field diary and a questionnaire applied to 46 workers from 9 companies in footwear, the results obtained show that workers

Cómo citar

Rojas-Caceres, Y.; Ordoñez-Ortiz, A.L. y Daza-Rios, C. T. (2021). Caracterización de prácticas de autocuidado y percepción del riesgo laboral en trabajadores de un grupo de empresas de calzado ubicadas en Bucaramanga, Colombia PostCOVID-19. Revista FACCEA, Universidad de la Amazonia, Vol. 11(2), 113-126. https://doi.org/10.47847/faccea.v11n2a1 
have a low level of self-care practices and a low level of risk perception. Therefore, it is necessary to generate strategies that increase self-care practices and risk perception in order to improve the well-being of workers.

Key words: work well-being, work condition, perception, health, lifestyle.

\section{INTRODUCCIÓN}

La mayoría de las empresas de calzado legalmente constituidas son MIPYMES (empresas micro, pequeñas y medianas). Estas cuentan con pocos empleados y desde las mismas hay un limitado interés por implementar sistemas de gestión de seguridad y de salud en el trabajo, por parte de los empleadores, debido a la inversión de tiempo y de los recursos necesarios que esto implica. Las MIPYMES poseen una mayor tasa de accidentalidad y una menor capacidad financiera, lo que las hace vulnerables a tener que disminuir o detener su producción al momento de tener que afrontar demandas, multas o sanciones, asociadas con una inadecuada gestión de los riesgos laborales, según lo mencionado por la Cámara Técnica de Riesgos Laborales (2018). A su vez, este es el tercer sector en generar el mayor aporte a la tasa de empleo de Bucaramanga, de acuerdo con lo que indica el Departamento Administrativo Nacional de Estadística y la Asociación Colombiana de Industriales del Calzado (DANE Y ACICAM, 2019); por consiguiente, es importante generar conocimiento y realizar estudios que promuevan la competitividad de las empresas del sector, y que además estimulen el bienestar laboral del alto número de trabajadores de este.

Ahora bien, el autocuidado y la percepción del riesgo son aspectos fundamentales para el correcto funcionamiento de las empresas, tal como lo expone Aldaz Arias (2018), en la investigación que versa sobre el autocuidado y sobre la cultura de la prevención, como herramientas de la salud laboral. En esta, a partir de la recopilación de información de expertos en el tema, se plantearon estrategias para el desarrollo de una cultura de autocuidado y de prevención del riesgo en el trabajo y, además, se resaltó que no basta con implementar prácticas como capacitaciones y utilizar los equipos especializados para cada trabajo, sino que además también es necesario promover estilos de vida saludables, así como una buena convivencia dentro y fuera del trabajo.

Mientras tanto, conforme con lo investigado por Gutiérrez y Pita (2015) acerca del diseño, documentación e implementación del sistema de salud ocupacional y seguridad industrial para la empresa calzado Leons, en el estudio realizado para la universidad de Santo Tomas, se evidencio, en el diagnóstico inicial a la empresa, que el cumplimiento de la normativa se encontraba en un 39\%, pero, dadas las características del sector, se entiende el nivel deficiente de cumplimiento y además la complejidad para mejorar ese cumplimiento; no obstante, después de la implementación, se pudo evidenciar la falta de importancia que se da al reporte de incidentes y de accidentes, lo cual puede impedir el mejoramiento en esta área, tras investigar la causa de los eventos laborales y ocasionar nuevamente su ocurrencia. 
A su vez, al igual que en la investigación anterior, a partir del estudio realizado por Osorio Gil (2016), el cual versa sobre el diseño e implementación de un sistema de seguridad y salud en el trabajo, en la empresa de calzado Queen Fashion, se encontró que las condiciones iniciales de la empresa eran muy bajas, en cuanto al cumplimiento de la normativa; sin embargo, existía un compromiso para incrementar el cumplimiento de esta. A partir de dicha investigación, también se encontró que es necesario tomar medidas especiales de intervención y de control, en las actividades correspondientes a los riesgos mecánicos, ya que estas son características recurrentes para el sector, y se demostró además que con la implementación de esto, hubo una mejoría en el desempeño de las tareas desarrolladas por los trabajadores, en condiciones seguras.

En otra investigación realizada por Jaimes Poveda (2019), desde la cual se estudia la incidencia de la implementación del sistema de gestión de seguridad y salud en el trabajo (SG-SST por sus siglas), en las empresas del sector calzado, teniendo en cuenta las variables de clima laboral, accidentabilidad y riesgos laborales, se observó que, después de la medición de las variables anteriormente mencionadas, estas podían influir en la productividad y en la reducción de gastos asociados con las demandas por accidentes y con las enfermedades de origen laboral. Tras definir los anterior, en dicho estudio también se obtuvo como resultado que las empresas que poseen un SG-SST, tienen mayor ventaja competitiva; también que cuentan con un mejor ambiente de trabajo; igualmente se estableció que los trabajadores presentan un mayor nivel de conciencia para ser parte de las actividades recreativas que beneficien su salud, lo cual permite tener bajas cifras de accidentabilidad y mejorar la calidad de vida de los trabajadores en general.

Así bien, de acuerdo con los resultados arrojados por las investigaciones antes mencionadas, con respecto a los beneficios para las organizaciones y para los colaboradores, se encuentra que es importante gestionar las prácticas de autocuidado y de percepción del riesgo en las empresas, ya que esto no solo es un requerimiento a cumplir, sino que también presenta beneficios para ambas partes. Sin embargo, para el sector calzado en el contexto de la ciudad de Bucaramanga, estas investigaciones se limitan a estudiar la gestión del SG-SST, sin realizar un estudio previo del estado en que se encuentran los trabajadores, con respecto a las prácticas de autocuidado y de percepción del riesgo. Teniendo en cuenta ese factor, en este estudio se buscó caracterizar dichos aspectos en los trabajadores de un grupo de empresas de calzado, que se encuentran ubicadas en la ciudad Bucaramanga, con el fin de generar conocimiento sobre este tema en dicho contexto, indicando análisis que pueda servir para definir medidas de intervención. Por su parte, la hipótesis para este proyecto, formulada con base en la investigación del estado del arte, es que los trabajadores de las empresas de calzado ubicadas en Bucaramanga no perciben los riesgos a los que están expuestos, al realizar las labores inherentes a su cargo y no cuentan con prácticas de autocuidado, siendo esta situación la causa de varios factores de riesgo. 


\section{METODOLOGÍA}

\section{Tipo de estudio}

La investigación que se desarrolló tuvo un alcance de tipo descriptivo-exploratorio, con un enfoque mixto y con un diseño no experimental de corte transversal.

\section{Participantes}

Trabajadores de un grupo de empresas de calzado ubicadas en Bucaramanga, Colombia. Las empresas seleccionadas como caso de estudio cuentan con las características propias de las compañías del sector de calzado.

\section{Materiales e instrumentos}

La muestra se seleccionó de manera no probabilística por conveniencia. La muestra de trabajadores pertenece a un grupo de empresas de calzado, las cuales están ubicadas en Bucaramanga, Colombia; estas cuentan con menos de 20 trabajadores y son consideradas MIPYMES.

\section{Unidad de análisis}

Trabajadores del grupo de empresas de calzado de Bucaramanga, Colombia.

\section{Técnicas e instrumento}

El instrumento de medición cuantitativo, sobre características sociodemográficas, prácticas de autocuidado y percepción del riesgo, fue validado por dos expertos; un experto en administración en salud ocupacional y un docente experto disciplinar en Seguridad y Salud en el Trabajo. La encuesta fue tomada y modificada de lo planteado por Espinoza Benavente (2017), agregando un cuestionario tomado de lesiones músculo esqueléticas de origen laboral (2014), con el fin de medir molestias músculo esqueléticas; también hubo apoyo a partir de la definición de peligros, del Instituto Colombiano de Normas Técnicas y Certificación (ICONTEC, 2010). Después de la validación se realizó la prueba piloto en campo, antes de proceder a recolectar la información; precedentemente a diligenciar el cuestionario, los participantes, de forma voluntaria, diligenciaron el consentimiento informado. Se utilizó también un diario de campo, en el cual se registraron las observaciones más relevantes y los comentarios de los trabajadores, sobre el concepto de accidente de trabajo; esta información se validó a partir de fuentes de información.

\section{RESULTADOS}

Como se puede observar en la Tabla 1 se encontró, de acuerdo con las características de edad, tiempo en el cargo y horas trabajadas, que la edad promedio de los trabajadores es de 38 a 76 años, lo que es coherente con el rango de edad más común entre los trabajadores, que es de los 31 a los 40 años; estos, laboran en promedio unas 9,67 horas al día. 
Tabla 1.

Variables edad, tiempo en el cargo y horas trabajadas.

\begin{tabular}{|c|c|c|c|c|c|c|c|c|}
\hline Factor & $\begin{array}{l}\text { Número de } \\
\text { trabajadores }\end{array}$ & $\%$ & $\%$ acumulado & Mínimo & Máximo & Media & Mediana & Moda \\
\hline Edad (Años) & 46 & - & - & 18 & 70 & 38,76 & 38 & 32 \\
\hline Menos de 20 años & 2 & 4 & 4 & - & - & - & - & - \\
\hline De 20 a 30 años & 9 & 20 & 24 & - & - & - & - & - \\
\hline De 31 a 40 años & 16 & 35 & 59 & - & - & - & - & - \\
\hline De 41 a 50 años & 11 & 24 & 83 & - & - & - & - & - \\
\hline Más de 50 años & 8 & 17 & 100 & - & - & - & - & - \\
\hline & 46 & - & - & 0,17 & 33 & & 11 & 10 \\
\hline \multicolumn{9}{|c|}{ Tiempo en el cargo (Años) } \\
\hline Menos de 1 año & 4 & 9 & 9 & - & - & - & - & - \\
\hline De 1 a 5 años & 13 & 28 & 37 & - & - & - & - & - \\
\hline De 5 a 10 años & 10 & 22 & 59 & - & - & - & - & - \\
\hline Más de 10 años & 19 & 41 & 100 & - & - & - & - & - \\
\hline & 46 & - & - & 4 & 14 & 9,67 & 10 & 8,10 \\
\hline \multicolumn{9}{|c|}{ Horas trabajadas al día } \\
\hline De 4 a 8 horas & 16 & 35 & 35 & - & - & - & - & - \\
\hline De 8 a 12 Horas & 28 & 61 & 96 & - & - & - & - & - \\
\hline Más de 12 horas & 2 & 4 & 100 & - & - & - & - & - \\
\hline
\end{tabular}

Fuente: elaboración propia de los autores.

En la Tabla 2 se puede observar que el cargo más frecuente entre los trabajadores es el de armador; la mayor parte de quienes ejercen este cargo son del sexo femenino; dichos trabajadores cuentan con un nivel de educación promedio de bachillerato finalizado; en mayoría, tienen un vínculo con la empresa de tipo verbal y gran parte de los contratos no suelen incluir el pago de prestaciones sociales. En los casos en los que el contrato sí incluye el pago de prestaciones sociales, la mayoría de los trabajadores solo cuenta con una afiliación a la entidad promotora de salud (EPS); aquellos, cuyo contrato no incluye el pago de prestaciones sociales, poseen una EPS subsidiada y solo dos cuentan con una administradora de riesgos laborales (ARL); ninguno es cotizante a una administradora de fondos de pensiones (AFP); lo anterior, debido a que la mayor parte de trabajadores no cuentan con un salario básico fijo y a que la modalidad de pago depende de las tareas realizadas y de los pares terminados, según los pedidos que deba cumplir la empresa. Así bien, durante temporada alta, la mayor parte de los trabajadores reciben un ingreso que oscila entre los $\$ 601.000$ a los $\$ 900.000$ COP o más; sin embargo, durante la temporada baja, la mayoría de los trabajadores reciben ingresos que van desde $\$ 0$ a $\$ 300.000 \mathrm{COP}$.

También se encontró que un amplio porcentaje de los trabajadores considera que nunca ha tenido un accidente de trabajo; solo 2 trabajadores mencionan haber tenido un único accidente de trabajo; además, la mayoría de los trabajadores afirma no tener un diagnóstico por enfermedad laboral y los 


\section{Tabla 2.}

Características sociodemográficas.

\begin{tabular}{|c|c|c|c|}
\hline \multirow{2}{*}{$\begin{array}{l}\text { Factor } \\
\text { Cargo }\end{array}$} & \multirow{2}{*}{$\frac{\text { de trabajadores }}{46}$} & \multicolumn{2}{|c|}{$\% \%$ acumulado } \\
\hline & & - & - \\
\hline Armador & $11 \quad 2$ & 23,9 & 23,9 \\
\hline Emplantillador & 4 & 8,7 & 32,6 \\
\hline Guarnecedor & 5 & 10,9 & 43,5 \\
\hline Administrador & 4 & 8,7 & 52,2 \\
\hline Cortador & 4 & 8,7 & 60,9 \\
\hline Decorador & 3 & 6,5 & 67,4 \\
\hline Solador & 6 & 13 & 80,4 \\
\hline Otros & 9 & 19,6 & 100 \\
\hline Sexo & 46 & - & - \\
\hline Mujer & 31 & 67 & 67 \\
\hline Hombre & 15 & 33 & 100 \\
\hline Pago de prestaciones sociales en el contrato & 46 & - & - \\
\hline $\mathrm{Si}$ & 15 & 33 & 33 \\
\hline No & 35 & 67 & 67 \\
\hline Prestación social con pagada por la empresa & 15 & - & - \\
\hline Eps & 14 & $93 \%$ & - \\
\hline AFP & 6 & $40 \%$ & - \\
\hline ARL & 5 & $33 \%$ & - \\
\hline Afiliación a Seguridad Social de tipo no Laboral & 31 & - & - \\
\hline No & 10 & $32 \%$ & - \\
\hline $\mathrm{Si}$ & 21 & $68 \%$ & - \\
\hline Prestación social con la que cuenta no pagada por la empresa & 21 & - & - \\
\hline EPS & 20 & 95,2 & - \\
\hline AFP & 0 & 0 & - \\
\hline ARL & 2 & 9,5 & - \\
\hline Tiene horario fijo de trabajo & 46 & - & - \\
\hline $\mathrm{Si}$ & 20 & 43 & 43 \\
\hline No & 26 & 57 & 100 \\
\hline $\begin{array}{l}\text { Enfermedades de condición hereditaria (pueden reportar más de } \\
\text { una enfermedad) }\end{array}$ & 46 & $\cdot$ & - \\
\hline Presión arterial & 16 & 35 & - \\
\hline Diabetes & 10 & 22 & - \\
\hline Cáncer & 3 & 7 & - \\
\hline Obesidad & 4 & 9 & - \\
\hline Mareos y Vértigo & 3 & 7 & - \\
\hline Colesterol & 7 & 15 & - \\
\hline Cardíacas & 2 & 4 & - \\
\hline
\end{tabular}

Fuente: Elaboración propia de los autores. 
Continuación Tabla 2.

Características sociodemográficas.

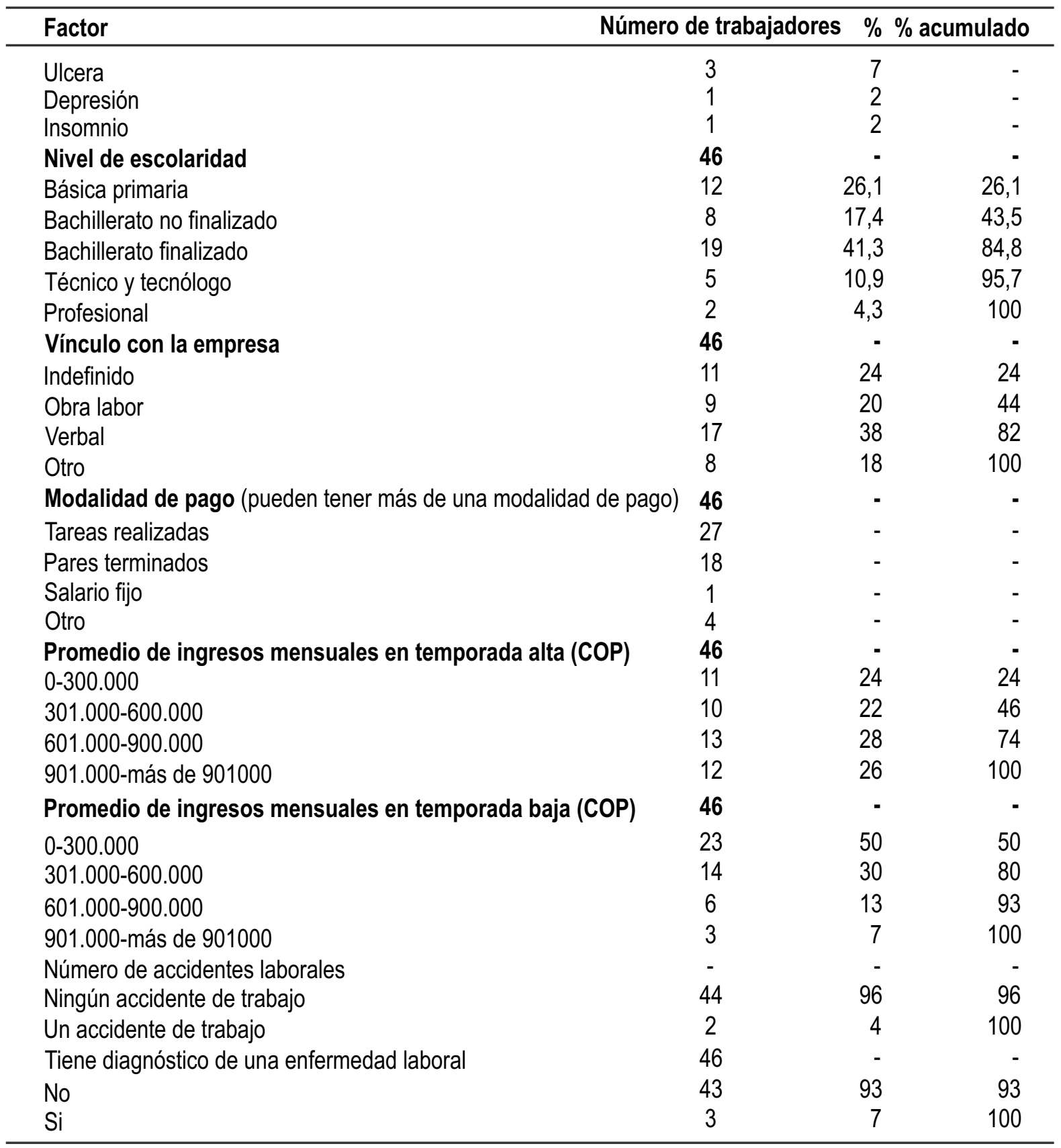

Fuente: Elaboración propia de los autores. 
3 que mencionaron tenerlo, no indicaron la enfermedad; por otra parte, en cuanto a enfermedades de condición hereditaria, las tres con mayor presencia entre los trabajadores fueron presión arterial, diabetes y colesterol.

Respecto de las prácticas de autocuidado, estas se evaluaron mediante una escala de Likert, y se presentaron como opción de respuesta las variables: nunca/casi nunca, algunas veces y casi siempre/siempre. Posteriormente se realizó una agrupación de las preguntas en cinco categorías, las cuales fueron: hábitos saludables no alimenticios; revisiones médicas; hábitos saludables en entornos laborales; hábitos saludables alimenticios y cuidados para prevenir el contagio de Covid19. Para clasificar el nivel de prácticas de autocuidado en el que se encontraban los trabajadores se estableció que, si el porcentaje del resultado se ubicaba del $0 \%$ al $60 \%$ de implementación de prácticas positivas de autocuidado, los trabajadores tenían un nivel bajo de autocuidado; respuestas en un rango del $60 \%$ al $85 \%$ de prácticas positivas de autocuidado se ubicaron en un nivel medio de autocuidado y respuestas con más del $85 \%$ de prácticas positivas de autocuidado, reportadas por parte de los trabajadores, tenían un nivel alto de autocuidado.

En la Tabla 3, se obtuvo como resultado que los trabajadores tenían un porcentaje de respuesta de prácticas positivas mayor, tanto para hábitos saludables no alimenticios como para cuidados para prevenir el contagio de Covid-19; este último, debido a la contingencia sanitaria que comenzó a inicios del año 2020. Por el contrario, las revisiones médicas y los hábitos saludables alimenticios fueron las prácticas que obtuvieron los puntajes más bajos.

Por otra parte, en el estudio de las molestias musculoesqueléticas, las cuales se midieron por medio de una escala de Likert, donde las opciones de respuesta eran nunca, a veces, muy a menudo y siempre, se encontró que el $78 \%$ de los trabajadores presentaba molestias musculoesqueléticas, en al menos una parte del cuerpo. Las partes del cuerpo se agruparon según el método de evaluación rápida de miembros superiores RULA, por sus siglas en inglés, el cual es mencionado en Lynn y Corllett (1993). En dicho estudio, las partes de cuerpo se dividen en dos grupos; el grupo A incluye: los brazos, los antebrazos y las muñecas; el grupo B abarca: el cuello, el tronco y las piernas. De las

Tabla 3.

Prácticas de autocuidado.

\begin{tabular}{lc}
\hline Prácticas de autocuidado & \% de respuestas de prácticas positivas. \\
\hline Hábitos saludables (No alimenticios) & 70 \\
Revisiones médicas & 42 \\
Hábitos saludables en entornos laborales & 55 \\
Hábitos saludables alimenticios & 46 \\
Cuidados para prevenir el contagio de Covid-19 & 75 \\
\hline
\end{tabular}

Fuente: Elaboración propia de los autores. 
partes anteriormente mencionadas, se presentaron mayor número de molestias en el cuello, la zona lumbar y la zona dorsal, para ambos sexos, incluyendo la muñeca derecha y la mano derecha en los hombres, mientras que las mujeres reportaron síntomas en las nalgas y/o en las caderas.

Por su parte, al evaluar la utilización de elementos de protección personal (EPP) se empleó una escala Likert, que tenía como opciones no lo conozco, nunca, casi nunca, algunas veces y siempre, para los (EPP) tapones de oídos, protectores de pantalla, gafas de seguridad, tapabocas reusable, tapabocas desechable, respiradores de filtro, guantes de maniobra, dedal de cuero y mandil de cuero. Se obtuvo además que los EPP con mayor porcentaje de uso fueron los tapabocas desechables y los tapabocas reusables; esto puede deberse, en gran medida, a que dichos elementos son utilizados para la prevención de contagio por Covid-19y, en tal sentido, como se pudo observar, estos hábitos de autocuidado son los más practicados por los trabajadores, sin que sea evidente el reporte específico de protección respiratoria pertinente para químicos aromáticos.

Respecto de la percepción del riesgo se hizo una evaluación mediante una escala de Likert, en la cual se tuvieron como opciones de respuesta: nunca/casi nunca, algunas veces y casi siempre/siempre. Posteriormente se clasificó cada una de las preguntas en los tipos de riesgos correspondiente, siendo estos biológicos, físico, químico, psicosocial, biomecánico, de condiciones de seguridad y fenómenos naturales. Para identificar el nivel de percepción del riesgo que tienen los trabajadores se estableció que, respuestas en el rango del 0 al 60\%, corresponden a que la percepción del riesgo está en un nivel bajo; del $60 \%$ al $85 \%$ se ubica en un nivel medio de percepción del riesgo y, para respuestas de más del $85 \%$, se definió que perciben el riesgo en un nivel alto. Como se puede observar en la Tabla 4, se evidenció que la mayoría de los trabajadores tienen un nivel medio de percepción del riesgo biomecánico y psicosocial; esto probablemente se presente porque tienen una sintomatología más notoria para dichos factores de riesgo. Por otra parte, se encontró que los trabajadores tienen un nivel bajo de percepción en cuanto a los riesgos biológicos, de condiciones de seguridad y físicos; esto es coherente con la falta de uso de EPP. Es de resaltar que aun en tiempo de pandemia, los trabajadores no percibieron como importante el factor de riesgo

Tabla 4.

Percepción del riesgo.

\begin{tabular}{lc}
\hline Riesgo & \% de respuestas de percepción del riesgo alta. \\
\hline Biológico & 27 \\
Físico & 38 \\
Químico & 31 \\
Psicosocial & 62 \\
Biomecánico & 79 \\
Condiciones de seguridad & 41 \\
Fenómenos naturales & 26 \\
\hline
\end{tabular}

Fuente: Elaboración propia de los autores. 
biológico en su trabajo y este fue considerado como el segundo más bajo junto con los fenómenos naturales.

\section{DISCUSIÓN DE RESULTADOS}

\section{Resultados de las características sociodemográficas}

El perfil de los trabajadores que se tuvieron en cuenta para esta investigación, en su mayoría corresponde a mujeres en edad adulta, ubicadas en el rango de los 31 a los 40 años, con Bachillerato finalizado y las cuales trabajan más de 9 horas al día sin prestaciones sociales incluidas en sus contratos y sin un salario fijo. Esto evidencia la vulnerabilidad y oportunidades de mejora de sus condiciones laborales.

Según lo encontrado en la investigación también se puede observar que, en su mayoría, los trabajadores reportan no haber tenido accidentes de trabajo y solo 2 mencionaron haber sufrido un evento laboral; sin embargo, gracias al diario de campo se observó que los trabajadores no tienen un concepto claro de accidente de trabajo, ya que comentaron no considerar la existencia de un accidente laboral, si este no requería de asistencia médica; ello también, en tanto consideraron que cualquier tipo de golpe, quemadura, corte, rozadura, etc., que no requiera asistencia médica, no era considerado por ellos como accidente. Por tanto, es necesario indicar que el número real de accidentes de trabajo que aquellos han tenido es incierto, pero se puede deducir que la cantidad es mucho mayor a la expuesta por los trabajadores.

\section{Resultados de prácticas de autocuidado}

Sobre las prácticas de autocuidado es evidente la necesidad de implementar actividades para promocionar las mismas en los entornos laborales y en especial lo relacionado con la realización de chequeos médicos preventivos y de hábitos saludables alimenticios, pero sin descuidar los hábitos saludables no alimenticios, ni las medidas de bioseguridad para prevenir el COVID-19.

Al comparar los resultados obtenidos para molestias musculoesqueléticas, con lo investigado por Vieira et al. (2015) y da Silva et al. (2017) y lo hallado en este proyecto se encontraron similitudes en las zonas del cuerpo que representan las molestias más frecuentes, las cuales son el cuello, la zona lumbar y la zona dorsal para ambos sexos. Sumado a esto, se encontró que los trabajadores de sexo masculino manifestaron también tener molestias en la muñeca y en mano derecha; por otro lado, las trabajadoras de sexo femenino manifestaron tener molestias en el área de las nalgas y de la cadera.

\section{Resultados de la percepción del riesgo laboral}

Los resultados de esta investigación son similares a los obtenidos por Ruiz García al. (2018), quien encontró que la utilización de los elementos de protección personal, EPP por sus siglas, fueron del $33 \%$, mientras que el porcentaje de quienes no hacen uso de estos fue del $67 \%$; esto es congruente con lo obtenido en el presente estudio, ya que los resultados fueron del $33 \%$ de utilización y del $56 \%$ 
de no utilización, además de un porcentaje de trabajadores que ni siquiera conoce los EPP sobre los que se les preguntaba, es decir, los tapones de oídos, los protectores de pantalla, las gafas de seguridad, los tapabocas reusables, los tapabocas desechables, los respiradores de filtro o los cartucho químicos, los guantes de maniobra y el dedal de cuero, mandil de cuero. Sin embargo, debido a la contingencia sanitaria causada por el Covid-19, se observó un aumento en el uso de tapabocas reusable y desechable ( $91 \%$ y $70 \%$ respectivamente), ya que esta es una de las principales medidas que se toman para prevenir el contagio; no obstante, se entiende que en circunstancias diferentes, estos porcentajes serían mucho más cercanos quizá, a los presentados por Ruiz García.

\section{CONCLUSIONES}

Teniendo en cuenta el perfil sociodemográfico de los trabajadores de un grupo de empresas de calzado se puede indicar que los estos se encuentran mayormente en el rango de edades entre los 31 y los 40 años; la mitad de dichos trabajadores tienen al menos una enfermedad de condición hereditaria, siendo las morbilidades más comunes la hipertensión, el colesterol y la diabetes, las cuales son clasificadas como enfermedades crónicas no trasmisibles. Lo anterior, muestra la importancia de promover un estilo de vida saludable en el entorno laboral, ya que los trabajadores pasan largas jornadas laborales en dicho entorno y, en tal sentido, ello resulta ser la mejor opción para la promoción de ese estilo de vida; a esto se suma la idea de que los trabajadores son vulnerables, ya que la mayoría no cuenta con una afiliación al sistema de seguridad social integral; por tanto, la combinación de hábitos no saludables, la morbilidad y la falta de protección pueden ocasionar consecuencias en los trabajadores y afectar su calidad de vida y de salud. Sin embargo, a pesar de todas estas condiciones adversas se encuentra que, en promedio, los trabajadores ejercen durante 10 años dichos cargos.

Las prácticas de autocuidado que son directamente responsabilidad del trabajador, como lo son los hábitos saludables no alimenticios, que comprenden las actividades físicas, el tiempo invertido en su salud, los cuidados, etc., se encuentran en un nivel medio, al igual que la de prevención de contagio de Covid-19. Se puede notar, sin embargo, que las empresas no promueven dichas prácticas, a pesar de que los trabajadores muestran estar interesados en ser capacitados acerca de estos temas; así mismo, asuntos como los hábitos saludables, incluidos los alimenticios, tanto como las revisiones médicas en entornos laborales se encuentran en un nivel bajo.

De manera general, se puede decir que los trabajadores poseen un nivel de percepción del riesgo medio-bajo, ya que solo los riesgos de tipo biomecánico y psicosocial tienen un nivel de percepción del riesgo medio; ello, probablemente porque estos riesgos inducen una sintomatología más fácil de identificar por parte de los trabajadores, en tanto los riesgos por fenómenos naturales, biológicos y físicos tuvieron una percepción baja del riesgo; igualmente, es de resaltar la baja percepción del riesgo biológico, aun en el contexto de la aparición de la pandemia por COVID-19. 
Se evidencia además que la prevención del contagio de Covid-19 fue la segunda practica implementada con mayor recurrencia, demostrando lo rápido que se adaptaron tanto los trabajadores como las empresas, a las prácticas de bioseguridad que se empezaron a implementar a inicios de marzo del 2020 en Colombia; en tal sentido, al momento de la realización de la encuesta, esto es, a mediados de agosto de 2020 e inicios de septiembre de ese mismo año, gran parte de los trabajadores ya aplicaban dichas medidas casi siempre/siempre. Contrario a esto, otras prácticas, las cuales llevan años siendo promovidas no son implementadas; sin embargo, se considera que la divulgación masiva a través de los medios de comunicación, sobre las actividades para evitar el contagio por COVID-19, pudo influir también en la transformación del comportamiento en el ambiente de trabajo

Finalmente, vale decir que la hipótesis planteada para este proyecto se aceptó, dado que, de manera general, los trabajadores tienen un nivel de autocuidado y de percepción del riesgo bajo; por lo tanto, se evidencia la necesidad de generar conocimiento sobre este tema en particular; también se requiere inversión, implementación e intervención de mejoras en el entorno laboral de los trabajadores, en tanto, de esta forma se promueve el bienestar de los mismos, así como la competitividad de la empresa, resultando esto en una relación de mutuo beneficio (Díaz-cabrera et al., 2008).

\section{Recomendaciones}

En cuanto a las prácticas de autocuidado se recomienda que las empresas inicien una gestión de estilos de vida saludables y de autocuidado en el entorno laboral, dado que la deficiencia de dichos estilos de vida no solo altera la salud de los trabajadores, sino también afecta a las mismas empresas, debido a que, por causa de estas enfermedades o padecimientos, se pueden presentar ausentismos, baja productividad o incluso multas; esto último, debido a que dichos programas deben ser implementados en las empresas, según el SG-SST.

En relación con la percepción del riesgo se recomienda capacitar a los trabajadores para que tengan conocimiento todos los riesgos a los que están expuestos, pero haciendo un especial énfasis en los que tienen un nivel bajo, como son los riesgos de tipo biológico, físico o químico; lo anterior, dado que de estos se pueden desprender enfermedades que en su mayoría son crónicas y por tanto, los trabajadores pueden no relacionarlas directamente con sus entornos laborales, en la medida en la que dichos padecimientos pueden ser multicausales; por lo tanto, los empleadores y los trabajadores pueden considerar que dichos riesgos no existen en sus entornos laborales. También se deben considerar los riesgos por fenómenos naturales; para ello se recomienda la implementación de planes de emergencia, dado que las instalaciones de las empresas no son óptimas para la evacuación adecuada de los trabajadores en caso de emergencia y que estas tampoco cuentan con señalizaciones ni con recursos necesarios para generar una respuesta adecuada. 


\section{Agradecimientos}

Especial agradecimiento a los dueños y trabajadores de las empresas de calzado, quienes permitieron el ingreso a los lugares de trabajo y a las empresas, y consintieron usar el tiempo de los trabajadores para responder el instrumento de medición.

\section{REFERENCIAS BIBLIOGRÁFICAS}

Aldaz A.L.L. A. (2018). Autocuidado y cultura de la prevención como herramientas de la salud laboral. [Tesis de grado, Pontificia Universidad Católica del Ecuador]. Recuperado de Repositorio institucional de PUCE. http://repositorio.puce.edu.ec/bitstream/handle/22000/15102/DISERTACIÓN\%2C ALDAZ ARIAS LILIANA LILIBETH.pdf?sequence=1\&isAllowed=y

Asociación Colombiana de Industriales del Calzado, el Cuero y sus Manufacturas (ACICAM). (2019, 14 de febrero). ¿Cómo va el sector? Enero a octubre de 2018. ACICAM. https://acicam.org/download/como-va-el-sector-octubre-2018/

Cámara Técnica de Riesgos Laborales. (2018). Seguridad y Salud en el trabajo: Una mirada desde la pequeña y mediana empresa.

https://www.ins.gov.co/seguridadysalud/docs/Memorias/9.pdf

Comisiones Obreras de Asturias (2014). Lesiones Musculoesqueléticas de Origen Laboral. http://tusaludnoestaennomina.com/wp-content/uploads/2014/06/Lesionesmusculoesqueléticas-de-origen-laboral.pdf

Da Silva, J. M. N., Da Silva, L. B., y Gontijo, L. A. (2017). Relationship between psychosocial factors and musculoskeletal disorders in footwear industry workers. Produção, 27, 1-13. https://doi.org/10.1590/0103-6513.231516.

Díaz- D., Isla-D., R., Rolo-G., G., Villegas-V., O., \& Hernández-F., Y.. (2008). La salud y la seguridad organizacional desde una perspectiva integradora. Papeles del Psicólogo, (29)(1), 83-91. http://www.redalyc.org/articulo.oa?id=77829110.

Espinoza B. M.C. (2017). Percepción de riesgo laboral y su relación con el Autocuidado en Profesionales de Enfermería de la Atención Primaria de Salud. [Tesis de Maestría-. Universidad de Concepción]. Recuperado de Repositorio Institucional UDEC. http://repositorio.udec.cl/bitstream/11594/2723/3/Tesis_Percepcion_de_riesgo_laboral.pdfGutié rrez P., C. M., y Pita B., L. N. (2015). Diseño, documentación e implementación de un sistema de salud ocupacional y seguridad industrial bajo los lineamentos de la norma NTC-OHSAS 18001: 2007 para la empresa calzado Leons en la ciudad de Bucaramanga. [Tesis de grado, 
Universidad Santo Tomas].

Hernández S., R., Fernández C., C., y Baptista L., P. (2014). Metodología de la investigación . McGRAW-HILL/ INTERAMERICANA EDITORES.

Instituto Colombiano de Normas Técnica y Certificación (ICONTEC). (2010). GTC-45: Guía para la identificación de los peligros y la valoración de los riegos en seguridad y salud ocupacional. (ICONTEC).

Jaimes P., A. (2019). Incidencia de la implementación del SG-SST en las variables clima laboral, accidentabilidad y riesgos laborales en las empresas del sector calzado [Tesis de maestría, Universidad cooperativa de Colombia]. https://doi.org/10.1017/CBO9781107415324.004

Lynn, M., y Corlett, N. (1993). RULA:A survey method for the investigation of work-related upper limb disorders. Applied Ergonomics, 24(2), 91-99.

Osorio G., C. M. (2016). Diseño e Implementación de un Sistema de Seguridad y Salud en el Trabajo ( SG-SST ) a partir del Decreto 1443 del 2014 en Calzado Queen Fashion.[ Tesis de grado, Universidad Santo Tomás]. Repositorio Institucional USTA. https://repository.usta.edu.co/bitstream/handle/11634/9455/OsorioGilCesarMauricio2016.pdf?s equence=1\&isAllowed=y

Ruiz G., L. K., Diaz G., N, Jiménez G., O. (2018, noviembre). Conocimientos y actitudes sobre el uso del equipo de protección personal en trabajadores del calzado de una fábrica de la ciudad de León, Guanajuato, México. (Ponencia) XVI Coloquio Panamericano de Investigación en En ferm ería. $c u b$. http://www.coloquioenfermeria2018.sld.cu/index.php/coloquio/2018/paper/view/503/421.

Vieira, E. R., Buckeridge S., M. V. G., Brentini de Almeida, L., Vieira V., W., Domingos S., J., y Veiga Q., P. R. (2015). Symptoms and risks for musculoskeletal disorders among male and female footwear industry workers. International Journal of Industrial Ergonomics, 48. 110-116. https://doi.org/10.1016/j.ergon.2015.05.001 